\title{
Diagnóstico y manejo actualizado del tromboembolismo pulmonar agudo
}

Diagnosis and updated management of acute pulmonary thromboembolism

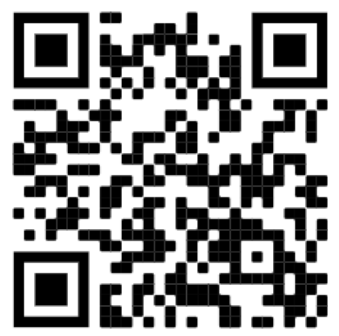

${ }^{1}$ Dr. Gustavo Piñar Sancho

Clínica Marcial Fallas, San José, Costa Rica

(D) https://orcid.org/0000-0002-1164-5483

${ }^{2}$ Dra. Vivian Abarca Zúñiga

Clínica Marcial Fallas, San José, Costa Rica

(D) https://orcid.org/0000-0002-2515-0238

${ }^{3}$ Dra. Sharon Moya Corea Investigadora independiente, San José, Costa Rica

(i) https://orcid.org/0000-0001-8941-7811

1 Médico general graduado en la Universidad Hispanoamericana (UH). cod. MED12320.

alonso0413@gmail.com

2 Médica general, graduada de la Universidad de ciencias médicas (UCIMED) cod.MED16024. viviaz17@hotmail.com

${ }^{3}$ Médica general, graduada de la Universidad de ciencias médicas (UCIMED) cod. MED11333.
CORREGIDO

$25 / 09 / 2020$
ACEPTADO

$27 / 08 / 2020$

\section{RESUMEN}

El tromboembolismo pulmonar es un padecimiento que se presenta con frecuencia de manera silenciosa, muchos de los casos no se diagnostican de forma oportuna. Es una complicación de la trombosis venosa profunda procedente, principalmente de los miembros inferiores, lo que provoca la oclusión total o parcial de la circulación pulmonar, ocasionada por un coágulo, que dependiendo de su magnitud, puede o no originar síntomas.

PALABRAS CLAVE: embolismo pulmonar; tromboembolismo; tromboembolismo venoso; terapia trombolítica; agentes fibrinoliticos.

\section{ABSTRACT}

Pulmonary thromboembolism is a disease that often occurs silently, many of the cases are not diagnosed in a timely manner. It is a complication of deep vein thrombosis originating, mainly in the lower limbs, which causes total or partial occlusion of the pulmonary circulation, caused by a clot, which depending on its magnitude, may or may not cause symptoms.

KEYWORDS: pulmonary embolism; thromboembolism; venous thromboembolism; thrombolytic therapy; fibrinolytic agents. 


\section{INTRODUCCIÓN}

El tromboembolismo pulmonar (TEP) es una enfermedad de dificil diagnóstico, y es un problema en el sistema de salud mundial. Es la tercera causa de muerte cardiovascular, tras la enfermedad coronaria y los accidentes vasculares cerebrales. No es una enfermedad que se pueda excluir con certeza sin la ayuda de estudios de imágenes de alto costo y del algunos examenes de laboratorio.

La tromboembolia venosa (TV) comprende la trombosis venosa profunda (TVP) y el tromboembolismo pulmonar (TEP), ocasiona muerte y discapacidad de origen vascular en todo el mundo. Cuando los trombos venosos profundos se desprenden de su sitio de formación, se transforman en émbolos para viajar a la vena cava, a la aurícula y al ventrículo derecho, para alojarse posteriormente en la circulación arterial pulmonar, y en ella causar el tromboembolismo pulmonar, provocando anomalias en el intercambio de oxigeno a nivel alveolar generando aumente del espacio muerto fisiologico y una disyuntiva en las unidades alveolo-capilares pulmonares.

Con lo anterior mencionado el presente artículo tiene como objetivo principal brindar una revisión bibliográfica sobre los aspectos más importantes del tromboembolismo pulmonar, diagnóstico y tratamiento adecuado actualizado, con el fin de proporcionar el conocimiento oportuno a los profesionales de la salud que se enfrentan a esta enfermedad.

\section{MÉTODO}

Para la elaboración del presente artículo tipo revisión bibliográfica se utilizaron 15 diferentes referencias bibliográficas las cuales se encuentran en idioma español e inglés. Dichas fuentes fueron publicadas entre los años 2012 y 2019. Las principales bases de datos utilizadas para la búsqueda de los diferentes artículos sobre el tema de interés fueron: Scielo, Uptodate, Elsevier, y PubMed y se emplearon como términos de búsqueda "Tromboembolismo Pulmonar", "Trombosis Venosa Profunda", "anticoagulación".

\section{EPIDEMIOLOGÍA}

La tromboembolia pulmonar (TEP) está considerada como una urgencia cardiovascular constituyendo una de las principales causas de morbi mortalidad, en pacientes hospitalizados. Se estima que la incidencia de la enfermedad a nivel mundial es de 100 a 200 casos por cada 100,000 habitantes. Tambien es causante de 317,000 muertes anuales en la union europea y el 59\% de las muertes relacionadas a TEP se hace el diagnóstico post mortem $(1,2)$.

Existen datos de algunos países en donde definitivamente el factor de riesgo más importante para embolia pulmonar es la presencia de trombosis venosa profunda y cuyo riesgo es mayor entre más proximal sea la obstrucción de los vasos de los miembros pélvicos (1).

\section{FISIOPATOLOGÍA}

La tríada de Virchow es el inicio del preceso protrombotico y comprende inflamación, hipercoagulabilidad y daño endotelial; culmina en el reclutamiento de plaquetas activadas que liberan micropartículas y estas últimas contienen mediadores proinflamatorios que se unen a neutrófilos, los estimulan para que liberen su material nuclear. Se forman trombos venosos y se 
multiplican en el entorno de estasis, baja presión de oxígeno y mayor cantidad de genes proinflamatorios. En el TEP agudo se afectan tanto la circulación como el intercambio de gases (2).

La primera causa de muerte por embolia de pulmón es debida al fracaso del ventrículo derecho por el aumento de presión en el mismo con desincronización de ambos ventrículos, lo que conlleva el fracaso del ventrículo izquierdo, una disminución de la fracción de eyección y la inestabilidad hemodinámica. La hipoxemia es secundaria al desajuste en la relación ventilación/perfusión debido a que existen zonas bien ventiladas pero mal perfundidas generando dificultad respiratoria (3).

\section{CLÍNICA}

La presentación clínica puede ser muy variable y presentarse desde un shock o hipotensión sostenida a disnea leve. Puede incluso ser asintomática y diagnosticarse mediante pruebas de imagen realizadas para otros fines. La gravedad de los síntomas depende principalmente de la magnitud del embolismo y la condición cardiorespiratoria previa. El síntoma más frecuente es la disnea, se manifiesta cuando el coágulo obstruye porciones proximales de la vasculatura pulmonar, puede manifestar irritación pleural y dolor tipo pleurítico hasta en $50 \%$ de los pacientes a veces acompañada de tos y hemoptisis. (4) Un número importante presentan también dolor retroesternal de tipo coronario isquémico, que puede confundir al clínico, éste es debido a sobrecarga aguda del ventrículo derecho y defectos de perfusión del mismo por aumento de la presión transmural.

El síncope es raro, pero es una presentación importante de TEP en un $10 \%$.
La presencia de shock cardiogénico generalmente obedecen a una tromboembolia pulmonar masiva ( $>40 \%$ de obstrucción de los vasos pulmonares). La exploración física tiene hallazgos variados dependiendo de la magnitud de la TEP. Por lo general los signos clínicos más frecuentes son taquicardia y taquipnea. La exploración pulmonar será normal, aunque en algunos pacientes puede haber estertores finos $y$ derrame pleural.

La evaluación de las extremidades inferiores es importante, en un $25 \%$ de los pacientes existen datos de trombosis venosa profunda; debe buscarse intencionadamente diferencia en los perímetros de ambas piernas $o$ dolor con las maniobras realizadas. La disnea, taquipnea y dolor torácico están presentes hasta en $97 \%$ de los enfermos con TEP y sin enfermedad cardiopulmonar previa (5).

\section{CLASIFICACIÓN}

\section{Tromboembolia pulmonar (TEP) de alto riesgo}

TEP agudo con hipotensión sostenida 0 shock (PAS menor a $90 \mathrm{mmhg}$ ) o que requiera uso de inotrópicos (sin asociación a otro diagnóstico cardiovascular que explique la condición hemodinámica del paciente). Presenta un riesgo relativo de mortalidad a 90 días de $32 \%(6)$.

\section{Tromboembolia pulmonar (TEP) de riesgo intermedio}

TEP agudo sin hipotensión sistémica pero con disfunción ventricular derecha y/o necrosis miocárdica.

Este subgrupo de pacientes amerita una estratificación por medio de la clasificación PESI (Pulmonary Embolism Severity Index) con medición de cámaras por 
ecocardiografía o tomografía contrastada, enzimas cardiacas y proBNP.

Según los hallazgos de dicha estratificación se puede definir un grupo de riesgo intermedio bajo y otro con riesgo intermedio alto, en el cual, a pesar de tener un riesgo de mortalidad a corto plazo de un $2 \%$, el riesgo de descompensación hemodinámica y de repercusión cardiopulmonar crónica es considerable y por ende se favorece un procedimiento de reperfusión. La implicación es que en este grupo de pacientes, la indicación de disminución de la mortalidad, parece ser sustituida por la necesidad de evitar posibles efectos adversos secundarios como la persistencia en la disfunción del ventrículo derecho, la hipertensión pulmonar crónica y el impacto en la calidad de vida (6).

\section{Tromboembolia pulmonar (TEP) de bajo riesgo \\ TEP agudo en ausencia de marcadores clínicos de pronóstico adverso que definan un TEP de alto riesgo o riesgo intermedio (6).}

\section{DIGNÓSTICO}

No existe ninguna prueba aislada que nos ayude a confirmar o descartar el TEP; es por ello que su diagnóstico se basa en varios pilares: sospecha clínica, datos analíticos y prueba de imagen. Al existitr la sospecha clínica, existen hoy en día escalas clínicas (Wells o Ginebra) (TABLA 1) ampliamente validadas y estandarizadas que podemos utilizar como primer paso al intentar diagnosticar una persona con TEP en los servicios de emergencias.

En caso que el valor de la escala de un valor de riesgo alto, se procede con la realización del dimero $D$, esto en el caso de que el paciente se encuentre hemodinamicamente estable (7).
En el FIGURA 1 se observa un esquema actualizado de toma de desiciones si se sospecha de TEP.

Existen otros metodos menos sensibles y menos especificos que podrían generar alguna sospecha en caso de ser realizados, pero no deben ser utilizados como examenes de rutina cuando se sospecha TEP en los pacientes.

- Gases arteriales: se esperaria encontrar hipoxemia, hasta el $40 \%$ tienen gasometría normal, a menudo se asocia con hipocapnia y alcalosis respiratoria (8).

- Radiografía de tórax: suele no estar alterada, pero estas alteraciones no suelen ser específicas, podemos encontrar atelectasias, derrames, etc, una radiografía normal no excluye el diagnóstico y sirve para descartar otras causas de dolor torácico o disnea. Entre las anomalías definidas están la oligemia focal (signo de Westermark); densidad cuneiforme periférica por arriba del diafragma (giba de Hampton) y agrandamiento de la arteria pulmonar descendente derecha (signo de Palla) (8).

- Electrocardiograma: se puede encontrar desde electrocardiogramas (ECG) sin alteraciones, a una taquicardia sinusal en los casos leves; un hallazgo de fibrilación auricular no conocida puede hacer sospechar la existencia de TEP; otro hallazgo en casos más graves es el conocido como patrón S1Q3T3, con bloqueo completo de rama derecha o inversión de la onda T en V1-V4 (9). 
FIGURA 1. Diagnóstico de tromboembolismo pulmonar agudo

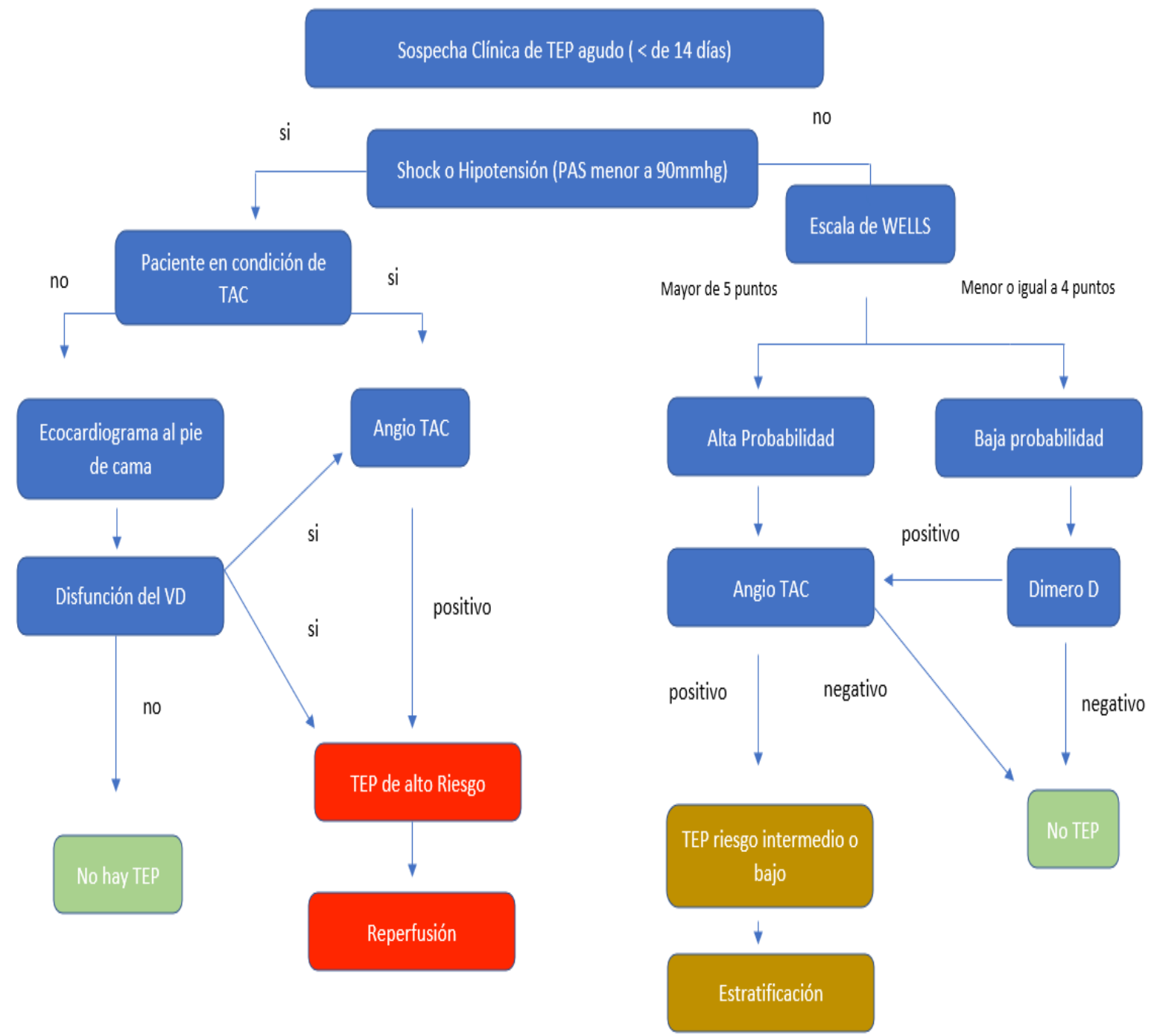

Fuente: A.Castuera. J. Fernandez. Trombo Embolismo Pulmonar, servicio Urgencias, Hospital Universitario Gregorio Marañon, Madrid España. 2015: 11 (88) pág 5245

- Troponina T-I: la elevación en plasma indica peor pronóstico y se ha asociado con una mayor mortalidad, mientras que valores bajos indican un buen pronóstico y pocas complicaciones a largo plazo; un elevado valor predictivo negativo es un dato analítico indispensable en el TEP como el NT-proBNP (10).
- NT-proBNP: se eleva en casos de inestabilidad hemodinámica, suelen indicar gravedad del TEP e indirectamente disfunción del ventrículo derecho. Niveles elevados indican un peor pronóstico (10).

- Dímero-D: es un producto de degradación de la fibrina presente en el 
trombo que se genera cuando este se rompe por la acción de la plasmina. Es una prueba de alta sensibilidad y baja especificidad, con alto valor predictivo negativo.

Por lo tanto un dímero $D$ negativo excluye el TEP en pacientes con probabilidad clínica baja. Existe evidencia actual que sugiere que el punto de corte del dímero-D debe ajustarse por edad en pacientes sobre 50 años. Si el punto de corte es $500 \mathrm{mcg} / \mathrm{L}$, se debe multiplicar por la edad por 10 (es decir a los 60 años el valor normal es 600 $\mathrm{mcg} / \mathrm{L}$ y a los $75,750 \mathrm{mcg} / \mathrm{L})$. en el momento que el hay una sospecha alta de TEP por medio de la escala de wells y ademas un valor de dimero $D$ alto para la edad, en ese momento se necesita un estudio de imagnes confirmatorio de TEP (11).

- Angiotomografía Computada de Tórax Helicoidal Multicorte (angio-TAC): es la prueba de elección para el diagnóstico de TEP, actualmente, la angio-TAC sería un método de elección por su alta sensibilidad (94\%), especificidad (100\%) y valor predictivo negativo (99\%). Genera todas las imágenes de tórax con $\leq 1 \mathrm{~mm}$ de resolución durante un lapso breve de apnea.

Es posible visualizar ramas de sexto orden con una resolución mejor que la de la angiografía corriente (12).

- Gammagrafía ventilación/perfusión: actualmente esta prueba ha sido desplazada por la angio-TC multicorte y se reserva para los pacientes con alergia al contraste; embarazadas, pacientes con mieloma (12).
- Ecocardiograma: no es un método de rutina para el diagnóstico del TEP; se puede realizar a pie de cama del paciente si su inestabilidad impide el traslado del paciente a la sala de rayos; un ecocardiograma sin datos de compromiso o disfunción de ventrículo derecho descarta el TEP como causa de dicha inestabilidad. El signo indirecto más conocido de TEP en la ecocardiografía transtorácica es el signo de McConnell: hipocinesia de la pared libre del ventriculo derecho con movimiento normal o hipercinético del vértice del ventriculo derecho (13).

\section{ESTRATIFICACION DE RIESGO}

Es necesario clasificar el riesgo de mortalidad de TEP precozmente durante la evaluación. Alrededor de $70 \%$ de los TEP son de bajo riesgo y actualmente hay evidencia que sugiere que pueden ser manejados en forma ambulatoria. El riesgo de mortalidad en TEP lo confiere fundamentalmente la presencia de falla del ventrículo derecho.

La primera línea de evaluación de riesgo es la clínica. La escala más utilizada es el puntaje Pulmonary Embolism Severity Index (PESI) y PESI simplificada, que incorpora comorbilidades y signos clínicos al momento de la evaluación inicial (TABLA 2).

Divide a los pacientes en 5 grupos de riesgo según puntaje total, en caso de la clasificación simplificada los clasifica solo en riesgo alto y bajo. Aquellos pacientes con un puntaje igual o mayor a 1 presenta un riesgo de mortalidad a 30 días de $11 \%$ (14).

La segunda línea de estratificación de riesgo es la disfunción de ventrículo derecho por medio de bioquímica, donde el pro-BNP y las 
troponinas han demostrado tener un valor predictor de complicaciones, recurrencia y mortalidad.

Finalmente, una relación alta de diámetros de ventrículo derecho/ventrículo izquierdo (VD/VI), medidos en el angioTAC de tórax, es otro marcador de mal pronóstico validado en TEP. Por lo tanto un paciente con diagnóstico de TEP, pero con un puntaje clínico de PESI bajo, con pro-BNP, troponinas normales y una relación VD/VI menor a $1 \mathrm{~cm}$, tiene bajo riesgo de mortalidad y puede iniciar anticoagulación efectiva $y$ manejarse en forma ambulatoria precozmente con un control estrecho. Si el paciente que se hospitalizó sin criterios de TEP de bajo riesgo (PESI alto, elevación de proBNP y/o troponina o dilatación de VD en angioTAC de tórax) no tiene criterios de disfunción de ventrículo derecho en ecocardiografía y no desarrolla compromiso hemodinámico en las primeras 24 - 48 horas de evolución, se clasifica como TEP de riesgo intermedio. De éste grupo un 5\% evoluciona con hipotensión y shock, es decir, TEP de alto riesgo (15).

\section{TRATAMIENTO}

- Anticoagulación: todo paciente con un diagnóstico de TEP agudo, salvo que presente alguna contraindicación, debe ser anticoagulado de forma apropiada y rápida con heparina no fraccionada o heparina de bajo peso molecular. El esquema clásico de inicio de terapia en TEP es de heparina no fraccionada (HNF). El empleo de HNF intravenosa debe comenzarse con un bolo de 80 UI/kg o $5.000 \mathrm{UI}$, seguido de una infusión continua intravenosa de $18 \mathrm{U} / \mathrm{kg} /$ hora 0 $1.300 \mathrm{Ul} /$ hora, con ajuste de la dosis de acuerdo con el tiempo de tromboplastina parcial activado (TTPA). El tratamiento con HNF debe ser mantenido, como mínimo, 5-7 días, para conseguir 1,5 a 2 veces el tiempo control de TTPA. En el caso de la heparina de bajo peso molecular (HBPM) que seria otra alternativa que presenta otras notables ventajas frente a la HNF. La HBPM no requiere monitorización, presenta menos incidencia de trombocitopenia inducida por heparina (TIH) y al poder administrarse por vía subcutánea permite completar el tratamiento en domicilio, ahorrando costos y mejorando la calidad de vida del paciente. No obstante, la HBPM debe administrarse con cautela en pacientes con insuficiencia renal y requiere monitorización (concentración de antiXa).

Por otro lado, se recomienda realizar un control de plaquetas entre el cuarto y séptimo día de tratamiento con HBPM, igual que con las HNF, para la detección de una posible $\mathrm{TIH}$, la dosis usal de la enoxaparina es de $1 \mathrm{mg} / \mathrm{kg}$ de peso dos veces al día, si el paciente tiene función renal normal (16).

El Fondaparinux es un inhibidor selectivo del factor Xa, constituye una alternativa a las HBPM.

Se administra por vía subcutánea en dosis ajustadas por el peso corporal. Debido a su vida media, 15-20 horas, fondaparinux se puede administrar una vez al día. Una ventaja potencial de este fármaco es que no se asocia a TIH y por tanto, no es necesario realizar un recuento plaquetario cuando se utiliza este fármaco (17).

Seguida de la anterior terapia escogida se debe administrar en forma de traslape un fármaco cumarínico (warfarina Vía oral), reduciendo la mortalidad por TEP de $25-30 \%$. 
TABLA1. Criterios modificados y simplificados de WELLS, probabilidad de TEP

\begin{tabular}{|l|c|}
\hline \multicolumn{1}{|c|}{ Criterios } & Puntos \\
\hline Síntomas y Sinos Clínicos de TVP & 3 \\
\hline Dignóstico alternativo menos probable que TEP & 3 \\
\hline Frecuencia Cardiaca mayor a 100 por minuto & 1.5 \\
\hline Inmovilización Prolongada & 1.5 \\
\hline Cirugias en las últimas 4 semnas & 1.5 \\
\hline TVP o TEP previos & 1.5 \\
\hline Hemoptisis & 1 \\
\hline $\begin{array}{l}\text { Cáncer en tratamiento actual o menos de meses, o en } \\
\text { cuidados paliativos }\end{array}$ & 1 \\
\hline \multicolumn{1}{|c|}{ Riesgo } & Menor 4 \\
\hline Baja & Mayor o igual a 4 \\
\hline Alta & \\
\hline Fuente: J. Sandoval. Diagnóstico y Tratamiento del Tromboembolismo pulmonar. Revista colombiana de medicina. 2015: 26(3), pág 339 \\
\hline
\end{tabular}

La warfarina no debe utilzarse como monoterapia porque aumenta el riesgo trombótico los primeros días, la dosis inicial es de $5 \mathrm{mg}$ al día, ajustando la dosis según el margen de INR (International Normalized Ratio) que debe estar en un rango entre 2-3 para determnar que la anticoagulación es óptima y segura para el paciente.

La duración de la anticoagulación recomendada en un primer episodio es de al menos 3 meses, es caso de ser una recurrencia de TEP se recomienda una anticoagulación extendida de 1 año o de por vida dependiendo de cada caso. Existen una nueva generación de anticiagulantes orales que solo se van a mencionar, con notables ventajas para el paciente, por ejemplo Edoxabán, Dabigatrán, Apixabán, Rivaroxabán que depende del país puede haber baja disponibilidad para un tratamiento extendido.

- Tratamiento trombolítico: el tratamiento trombolítico resuelve rápidamente la obstrucción tromboembólica y tiene efectos beneficiosos en los parámetros hemodinámicos.

La trombolisis consti-tuye el tratamiento de primera elección en pacientes con TEP e inestabilidad hemodinámica, definido por la presencia de falla del ventriculo derecho o hipotensión arterial persistente (presión arterial media menor a $65 \mathrm{mmHg}$ ) no causada por una arritmia de comienzo reciente, hipovolemia o sepsis. 
TABLA 2. Escala clínica pronóstica en pacientes con tromboembolismo pulmonar agudo (TEP): Pulmonary Embolism Severity Index (PESI)

\begin{tabular}{|l|c|c|}
\hline \multicolumn{1}{|c|}{ PARÁMETROS } & PESI & PESI simplificada \\
\hline Edad & Mayor a 80 años & Mayor a 80 años \\
\hline Sexo varón & 30 & - \\
\hline Cáncer & 10 & 1 \\
\hline Insufucuencia cardiaca & 10 & 1 \\
\hline $\begin{array}{l}\text { Enfermedad Pulmnar Obstructiva } \\
\text { Crónica }\end{array}$ & 20 & 1 \\
\hline $\begin{array}{l}\text { Frecuencia cardiaca }>110 \text { por } \\
\text { min }\end{array}$ & 30 & - \\
\hline Presión arterial < 100mmhg & 20 & - \\
\hline $\begin{array}{l}\text { Frecuencia respiratoria }>0 \text { igual } \\
\text { a 30 por minuto }\end{array}$ & 20 & - \\
\hline Temperatura $<36$ C & 60 & 1 \\
\hline Estado mental alterado & 20 & \\
\hline Saturacion $<90 \%$ & & \\
\hline
\end{tabular}

Estretificación de Riesgo

Clase 1 riesgo bajo < 65 puntos

Clase 2 riesgo bajo 65-85 putos

Riesgo bajo: 0 puntos

Clase 3 riesgo intermedio 86-105 puntos

Clase 4 riesgo alto $106-125$ puntos

Clase 5 riesgo muy alto $>125$ puntos

Riesgo alto: mayor o igual a 1

punto

Fuente: J. Sandoval. Diagnóstico y Tratamiento del Tromboembolismo pulmonar. Revista colombiana de medicina. 2015: 26(3), pág 339

Los trombolítocos mas usados se ejemplifican en la TABLA 3 (18).

- Filtros de vena cava inferior: las dos indicaciones principales para colocar el filtro en vena cava inferior son:
1) hemorragia activa que impide el uso de anticoagulantes.

2) trombosis venosa recurrente a pesar de la anticoagulación intensiva. 
TABLA3. Principales agentes trombolíticos

\begin{tabular}{|l|l|}
\hline rtPA o alteplasa & $\begin{array}{l}10 \mathrm{mg} \text { en bolo, seguido de } 90 \mathrm{mg} \text { iv en perfusión en } 1-2 \\
\text { horas; otra opción: } 0,6 \mathrm{mg} / \mathrm{kg} \text { en } 15 \mathrm{~min} \text { (máx } 50 \mathrm{mg})\end{array}$ \\
\hline Uroquinasa & $\begin{array}{l}4.400 \mathrm{Ul} / \mathrm{kg} \text { durante } 10 \mathrm{~min} \text {, seguido de } 4.400 \mathrm{UI} / \mathrm{kg} / \mathrm{hora} \\
\text { durante } 12 \text { horas }\end{array}$ \\
\hline Estreptoquinasa & $\begin{array}{l}5.000 \mathrm{UI} \text { en } 30 \mathrm{~min}, \text { seguido de } 100.000 \mathrm{UI} / \mathrm{h} \text { en } 12-24 \text { horas } \\
1,5 \text { millones Ul durante } 2 \text { horas }\end{array}$ \\
\hline
\end{tabular}

Fuente: A.Castuera. J. Fernandez. Trombo Embolismo Pulmonar, servicio Urgencias, Hospital Universitario Gregorio Marañon, Madrid España. Medicine. 2015;11(88):5250 pag

- Tratamiento endovascular en TEP: está indicado en TEP de alto riesgo con contraindicación absoluta o relativa a la trombolisis sistémica, o en pacientes que fueron sometidos a trombolisis sistémica, pero no logran recuperar el estatus hemodinámico ni ventilatorio.

El objetivo de intervención percutánea es remover o fragmentar el trombo obstructivo de la arteria pulmonar principal y así, disminuir la resistencia vascular pulmonar (la post carga del ventrículo derecho) y recuperar la función ventilatoria y el gasto cardíaco (19).

- Fragmentacion de trombos: consiste en desorganizar el trombo y dividirlo en fragmento de menor tamaño, esto puede se realizar en forma manual con catéteres como pigtail o con balones que dilatan la zona donde esta el trombo. La desventaja de esta técnica es que desencadena macroembolías y podrían desencadenar mayor deterioro hemodinámico.

- Trombectomia reolítica: es un catéter que inyecta solución salina a presión a nivel del trombo y genera, por el principio de Bernoulli, remoción de fragmentos de trombos (Angiojet®).

Los efectos adversos descritos son bradicardia y hemoglobinuria secundaria a hemólisis que habitualmente es reversible (19).

- Embolectomia por succión: es la extracción de trombos por succión, puede ser manual con catéter de lúmenes 8-9F aplicando presión negativa con jeringas de $60 \mathrm{ml}$, inconveniente de esta técnica es la hipovolemia y la anemia asociada a cada succión.

- Trombolisis guiada por cateter en TEP: las intervenciones endovasculares para el TEP pueden usarse como una opción cuando existen ciertas contraindicaciones a la trombolisis sistémica o como una alternativa mínimamente invasiva a la clásicamente las terapias endovasuculares involucran alguna dispositivo para disminuir la carga de trombo a nivel del árbol vascular pulmonar, ya sea por fragmentación, aspiración o terapia reolítica.

Dichas modalidades se pueden combinar con una terapia de infusión de trombolítico guiada por catéter, lo que implica menores tiempos y dosis de 
infusión, o pueden utilizarse en una modalidad meramente mecánica, como en aquellos casos donde exista una contraindicación al uso de trombolíticos y en donde el riesgo quirúrgico de una embolectomía pulmonar sea considerable (20).

En el año 2014, fue autorizado el uso de la terapia de fibrinolisis guiada por catéter de baja dosis guiada con ultrasonido (conocida como terapia con catéter EKOS), para el tratamiento de TEP. Dicha terapia implica la infusión intratrombótica de alteplase a bajas dosis (en un rango estudiado de 4 a 24 mg de dosis total por paciente) y por corto tiempo (rango estudiado de 2 a 24 horas). El catéter encargado de la infusión, a su vez posee varios dispositivos que emiten ondas de radiofrecuencia de baja amplitud que logran romper los enlaces de las cadenas de fibrina y a su vez aumentan la penetración del trombolítico dentro del trombo (20).

- Embolectomía pulmonar: se recomienda el uso de la embolectomía pulmonar en pacientes con TEP de alto riesgo y riesgo intermedio alto, para pacientes con contraindicación absoluta para la trombolisis, pacientes que ameritan la remoción de un trombo atrial derecho y aquellos pacientes en donde a pesar de un protocolo adecuado, la trombolisis no ha sido efectiva. Se reporta una mortalidad y morbilidad que rondan el $3 \%$ y $29 \%$ respectivamente (20).

\section{CONCLUSIÓN}

El tromboembolismo pulmonar es una de las enfermedades más incapacitantes del mundo, es por esto que debe ser abordada de manera precoz, ya que sus síntomas pueden avanzar hasta la muerte.

Se debe realizar una historia clínica adecuada para poder identificar cualquier causa médica, además de esto se pueden utilizar escalas de tamizaje como la de Wells, que permiten establecer la probabilidad real de un tromboembolismo pulmonar agudo, y luego se debe verificar el diagnóstico mediante estudios especializados como el angio TAC.

En los últimos años ha cambiado un poco el abordaje terapéutico de esta enfermedad, en especial si el paciente presenta un TEP de riesgo moderado o un TEP de riesgo alto, debido a lo agresivo que debe ser el manejo de estos pacientes para evitar complicaciones mayores. Los avances tecnológicos y estudios recientes han ayudado que el ultrasonido al pie de la cama del paciente grave sea una herramienta muy utilizada para un diagnostico rápido de dicha patología si el paciente no puede ser llevado a una sala de TAC por la gravedad de su sintomatología.

El tiempo de tratamiento de un primer episodio se debe extender aproximadamente 6 a 12 meses, antes de hablar de falla terapéutica. Existen fármacos que no son tan bien tolerados por el paciente con TEP, por lo que es posible probar las distintas alternativas para hallar la terapia individualizada óptima.

El tratamiento médico con anticoagulantes modernos ha demostrado una gran eficacia sobre todo en los pacientes con TEP de bajo riesgo que pueden ser manejados de manera ambulatoria para así no tener que estar internado en un centro hospitalario además de que se ha demostrado en algunos estudios el bajo riesgo de muerte, se debe tener en cuenta que no es para todo 
paciente, pero con una adecuada selección existe una gran posibilidad de remisión.

En este tema queda mucho por explorar, ya

que la industria médica ha mejorado la forma en que se diagnostica, estratifica y se maneja el tromboembolismo pulmonar agudo.

\section{REFERENCIAS}

1. Uresandi F, Monreal M, Gracía-Bragado F, Domenech P, Lecumberri R, Escribano P, et al. Consenso nacional sobre el diagnóstico, estratificación de riesgo y tratamiento de los pacientes con tromboembolismo pulmonar. Arch Bronconeumol. 2013. https://doi.org/10.1016/j.arbres.2013.07.008

2. Kucher et al. Randomized, Controlled Trial of Ultrasound-Assisted Catheter-Directed Thrombolysis for Acute intermediate Risk Pulmonary Embolism. Circulation. 2014;129: páginas 479-486. https://doi.org/10.1161/CIRCULATIONAHA.113.005544

3. Piazza et al. A Prospettive, Single-Arm, Multicenter Trial of Ultrasound-Facilitated, Catheter- Directed, Low-Dose Fibrinolysis for Acute Massive and Submissive Pulmonary Embolism. J Am Coll Cardiol Intv. 2015;8:2382-92. https://doi.org/10.1016/i.jcin.2015.04.020

4. Tapson et al. A Randomized Trial of the Optimum Duration of Acoustic Pulse Thrombolysis Procedure in Acute Intermediate Risk Pulmonary Embolism. J Am Coll Cardiol Intv. 2018;11: páginas 1401-1410. https://doi.org/10.1016/i.jcin.2018.04.008

5. Kearon C, Akl EA, Comerota AJ, et al. American College of Chest Physicians. Antithrombotic therapy for VTE disease: antithrombotic therapy and prevention of thrombosis. 9th edition: American college of chest physicians evidence based clinical practice guidelines. Chest 2012;141(Suppl 2): páginas 419-494. https://doi.org/10.1378/chest.141.5.1369b

6. Fernando Quiros Mata. PROTOCOLO DE MANEJO MULTIDISCIPLINARIO DEL TROMBOEMBOLISMO PULMONAR AGUDO. Hospital Ssan Juan De Dios. Caja Costarricense Del Seguro Social Agosto 2019 pag 1 19. Revizado en agosto del año 2020

7. Wells PS, Forgie MA, Rodger MA. Treatment of Venous Thromboembolism. JAMA 2014, 311:717-728. https://doi.org/10.1001/jama.2014.65

8. Kearon C, Akl EA, Comerota AJ, et al. American College of Chest Physicians. Antithrombotic therapy for VTE disease: antithrombotic therapy and prevention of thrombosis. 9th edition: American college of chest physicians evidence based clinical practice guidelines. Chest 2012;141(Suppl 2): páginas 419-494. https://doi.org/10.1378/chest.141.5.1369b

9. EINSTEIN-PE Investigators, Büller HR, Prins MH, Lensin AW, et al. Oral rivaroxaban for the treatment of symptomatic pulmonary embolism. N Engl J Med 2012;366: páginas 1287-1297 https://doi.org/10.1056/NEJMoa1113572

10. Agnelli G, Buller HR, Cohen A, et al. Oral apixaban for the treatment of acute venous thromboembolism. N Engl J Med 2013;369(9):799-80. https://doi.org/10.1056/NEJMoa1302507 
11. Clive Kearon, M.B., Ph.D., Kerstin de Wit, M.B., Sameer Parpia, Ph.D., Diagnosis of Pulmonary Embolism with dDimer Adjusted to Clinical Probability. The new england journal of medicine. nejm.org November 28, 2019 med 381;22. https://doi.org/10.1056/NEJMoa1909159

12. Buller HR, Davidson BL, Decousus $H$, et al. Subcutaneous fondaparinux versus intravenous unfractionated heparin in the initial treatment of pulmonaryembolism. $\mathrm{N}$ Engl J Med 2003;349: páginas 1695-702. https://doi.org/10.1056/NEJMoa035451

13. Rolf $P$. Engelberger and Nils Kucher. Catheter-Based Reperfusion Treatment of Pulmonary Embolism. Circulation 2011;124: 2139-2144 https://doi.org/10.1161/CIRCULATIONAHA.111.023689

14. A.I. Castuera Gil, J. Fernández Herranz, E. Martínez Larrull e I. Muñoz Roldán. Tromboembolismo pulmonar. Servicio de Urgencias. Hospital General Universitario Gregorio Marañón. Madrid. España. Medicine. 2015;11: páginas 5245-5253 https://doi.org/10.1016/j.med.2015.10.006

15. Jorge Sandoval B., Matias Florenzo V., Diagnostico y tratamiento del tromboembolismo Pulmonar. Rev.Med.Clin.Condes. 2015 pag 338-343 https://doi.org/10.1016/j.rmclc.2015.04.021

16. Ays segülS s,entürk*, EmineArgüder, ElifBabaog \u, HabibeHezeryH.CananHasanog lu. Diagnóstico por la imagen del tromboembolismo pulmonar mediante ecografía endobronquial. ELSEVIER DOYMA. Arch Bronconeumol. 2013;49(6):268-271 https://doi.org/10.1016/j.arbres.2012.08.006

17. Motta-Ramírez GA. Bastida-Alquicira J. Béjar-Cornejo Jr. Craviotto AB Salgado-Camarillo J. El ultrasonido y su papel preponderante en situaciones de urgencia. Anales de Radiología México 2014;13:404-427.

18. Sharif S, Eventov M, Kearon C, et al. Comparison of the age adjusted and clinical probability adjusted D-dimer to exclude pulmonary embolism in the emergency department. Am J Emerg Med 2019;37: 845-50. https://doi.org/10.1016/j.ajem.2018.07.053

19. Hendriksen JM, Geersing GJ, Lucas- sen WA, et al. Diagnostic prediction mod- els for suspected pulmonary embolism: systematic review and independent exter- nal validation in primary care. BMJ 2015; 351:h4438. https://doi.org/10.1136/bmj.h4438

20. Raja AS, Greenberg JO, Qaseem A, Denberg TD, Fitterman N, Schuur JD. Evaluation of patients with suspected acute pulmonary embolism: best practice advice from the Clinical Guidelines Com- mittee of the American College of Physi- cians. Ann Intern Med 2015;163: 701-11. https://doi.org/10.7326/M14-1772 